\title{
Do lifestyles influence the presence of promiscuous enzymes in bacteria and Archaea metabolism?
}

\author{
Mario Alberto Martínez-Núñez ${ }^{1^{*}}$ (1) and Ernesto Pérez-Rueda²
}

\begin{abstract}
The adequacy of an organism to the environment depends in part on its ability to modify its repertoire of cellular components, such as transporters, regulatory proteins and metabolic enzymes. In this review we discuss some recent results showing how the environment influences the content of promiscuous enzymes in bacterial and archaeal metabolism. In this regard, the proportion of promiscuous enzymes do not depend on genome size, as has been reported for the complete repertoire of enzymes, but it is influenced by the lifestyles, where the fraction of promiscuous enzymes is high in free-living organisms, while there is a small fraction in intracellular organisms. Therefore, promiscuous enzymes are enriched in organisms that inhabit fluctuating environments, providing bacteria with an enzymatic repertoire of new activities that helps to face multiple ecological variables. Finally, we discuss the possible role of gene duplications that occur most frequently in promiscuous enzymes of free-living organisms, which aid expand the universe of possible functions.
\end{abstract}

Keywords: Metabolism, Bacteria, Archaea, Comparative genomics, Duplicated enzymes

\section{Background}

The organisms contend and adapt to environment fluctuations using and modifying their repertoire of cellular components, such as transporters, regulatory proteins and metabolic enzymes. In general, the enzymes play a central role on these adaptive processes since they carry out the biochemical transformations of substrates into useful products, providing to cell with matter and energy. In classical terms, enzymes have been described as proteins catalyzing only one type reaction on specific substrates. However, diverse analyses have shown that some enzymes may play multiple physiological roles [1, 2]. These multifunctional enzymes, in turn, can be classified as moonlighting and promiscuous, according to whether domains that are present in the enzyme have catalytic activity or not $[1,3-5]$.

\footnotetext{
*Correspondence: maal.martinez@gmail.com

1 Laboratorio de Ecogenómica, Unidad Académica de Ciencias y

Tecnología, Facultad de Ciencias, Universidad Nacional Autónoma de

México, Mérida, Yucatán, Mexico

Full list of author information is available at the end of the article
}

In this minireview we discuss the recent results that suggest that the environment is shaping and maintaining the repertoire of promiscuous enzymes in the metabolism of bacteria and Archaea organisms. In the first section, the multifunctional enzymes and their classification in moonlighting and promiscuous are examined; later, the promiscuity present in enzymes, according if it is by substrate or catalytic, is also explored. Finally, we discuss some concerns about the evolution of promiscuous enzymes and the influence of environment and duplication process on them.

\section{Multifunctional enzymes reflect multiple functional roles}

Multi-functional enzymes have multiple physiological roles in the cell [6], and can be classified in moonlighting and promiscuous. Moonlighting proteins comprise a class of multifunctional proteins in which a single polypeptide chain performs multiple physiologically relevant biochemical or biophysical functions [7]. Moonlighting enzymes have a catalytic activity, and noncatalytic activity, which can be associated to regulation

\section{Springer}


or protein-protein interactions among other functions. These enzymes can carry out their functions in different structural domains [1], or in separate cell compartments, e.g. the bacterial cytoplasm or the cell surface [8]. This class of proteins have been described in different species from bacteria to humans, such as aconitase (EC: 4.2.1.3) of Homo sapiens (UniProt:Q71UF1), which catalyzes the isomerization of citrate to isocitrate, and has a complementary activity of iron-responsive elementbinding protein; or the biotin synthetase (EC: 6.3.4.15) BirA (UniProt:P06709) from the bacterium Escherichia coli with a secondary activity as transcriptional repressor of the bio operon, which is dependent on cellular concentration of biotin [9]. As a counterpart, promiscuous enzymes are composed of catalytic domains performing secondary biochemical reactions, not affecting the primary catalytic activity. Existence of promiscuity in enzymes has been linked to conformational diversity; namely, conformational changes enable the same enzyme to accommodate different substrates. In particular, the mobility of active site loops appears to play a key role in mediating promiscuity [10]. In this regard, enzyme promiscuity may be also explained because their active site can exclude substrates that are too far large to fit or with charges in place that cause repulsion between them; in contrast small substrates may fit into a capacious active site, although they may lose some of the binding affinity available to the optimal substrate. Even larger substrates may be able to bind if part of the molecule can protrude from the active site into the solvent. Thus, promiscuity may exist simply because it is impossible to exclude all potential substrates. Alternatively, promiscuity could be a relic of past activities in ancestral generalist enzymes that catalyzed more than one reaction [11].

\section{How many kinds of promiscuity are there?}

Promiscuous enzymes can present substrate or catalytic promiscuity. Substrate promiscuity has been described as the ability of enzymes to perform comparable chemical transformations using different substrates, which allow microbes to degrade different compounds [4, 12]. In this regard, the capability of an enzyme to process more than one substrate is intimately related to its evolvability, and promiscuous enzymes are more likely to appear uniformly distributed across species in the tree of life [13]. In contrast, catalytic promiscuous enzymes carry out a secondary reaction that results in a chemical transformation different from that catalyzed with its canonical substrate [11]. In this context, catalytic promiscuity may become less efficient than the primary reaction, such as the secondary phosphoserine phosphatase activity of HisB (UniProt:P06987) and
Gph (UniProt:P32662) enzymes from E. coli, whose $k_{\text {cat }} / \mathrm{K}_{\mathrm{M}}$ have values of 1.6 and $7.6 \mathrm{M}^{-1} \mathrm{~s}^{-1}$, respectively; whereas the $k_{\text {cat }} / K_{M}$ of the physiological phosphoserine phosphatase SerB (UniProt:P0AGB0) is of $8.7 \times 10^{4}$ $M^{-1} s^{-1}$ [14]. In other cases the promiscuous activities can be quite efficient, as in the enzyme homoserine kinase (ThrB, UniProt:P00547) from E. coli, which catalyzes the phosphorylation of homoserine with a $k_{\text {cat }} / \mathrm{K}_{\mathrm{M}}$ of $3.8 \times 10^{5} \mathrm{M}^{-1} \mathrm{~s}^{-1}$, while the promiscuous reaction of phosphorylation of 4-hydroxythreonine is done with a fairly robust $k_{\text {cat }} / \mathrm{K}_{\mathrm{M}}$ of $4.8 \times 10^{3} \mathrm{M}^{-1} \mathrm{~s}^{-1}$ [15]; or the arylsulfatase from Pseudomonas aeruginosa which catalyzes the primary reaction of hydrolysis of $p$-nitrophenyl sulfate with a $k_{\text {cat }} / \mathrm{K}_{\mathrm{M}}$ of $4.9 \times 10^{7} \mathrm{M}^{-1} \mathrm{~s}^{-1}$, whereas the secondary reaction of hydrolysis of bis(4-nitrophenyl) phosphate has a $k_{\text {cat }} / K_{\mathrm{M}}$ of $2.5 \times 10^{5} \mathrm{M}^{-1} \mathrm{~s}^{-1}$ [16]. These examples show that the rate constant for many promiscuous reactions are within the range of physiologically relevant reactions, which $k_{\text {cat }} / \mathrm{K}_{\mathrm{M}}$ round between $10^{3}$ and $10^{5} \mathrm{M}^{-1} \mathrm{~s}^{-1}$ as observed in metabolic enzymes [11]. Thereby, promiscuous enzymes could achieve diverse activities at the same protein, being one of them (in general) more efficient that the second one. This identification and characterization of promiscuous enzymes open a universe of proteins that can be used as a starting point for directed evolution of new biocatalysts for reactions with potential uses in pharmaceutical or biotechnological industries $[17,18]$.

\section{The content of microbial promiscuous enzymes is influenced by the environment}

A relevant question to be explored is how the metabolism and in particular the repertoire of promiscuous enzymes is impacted by the environment. With regard to this, recent studies have evidenced that the total fraction of enzymes in bacterial and archaeal genomes depends on a physical variable, the genome size, i.e. small genomes have a greater fraction of enzymes, while large genomes have a fewer fraction [19] (Fig. 1a). As a counterpart, promiscuous enzymes do not seem to have an evident correlation with genome size, remaining constant in all the organisms analyzed, Spearman's coefficient $=0.01$ (P value $=0.70)[20]$ (Fig. 1b). Indeed, recent studies have revealed that around $10 \%$ of the total enzymatic repertoire in bacteria and Archaea organisms correspond to promiscuous enzymes [19]. Therefore, the promiscuity represents a source that would provide a repertoire of different substrate and catalytic activities that can be used when the environment changes. This repertoire can be modified by the ecological conditions to which the bacteria face, i.e. chemical environment or substrate matrix, could influence the evolutionary trajectories of evolving enzymes; 


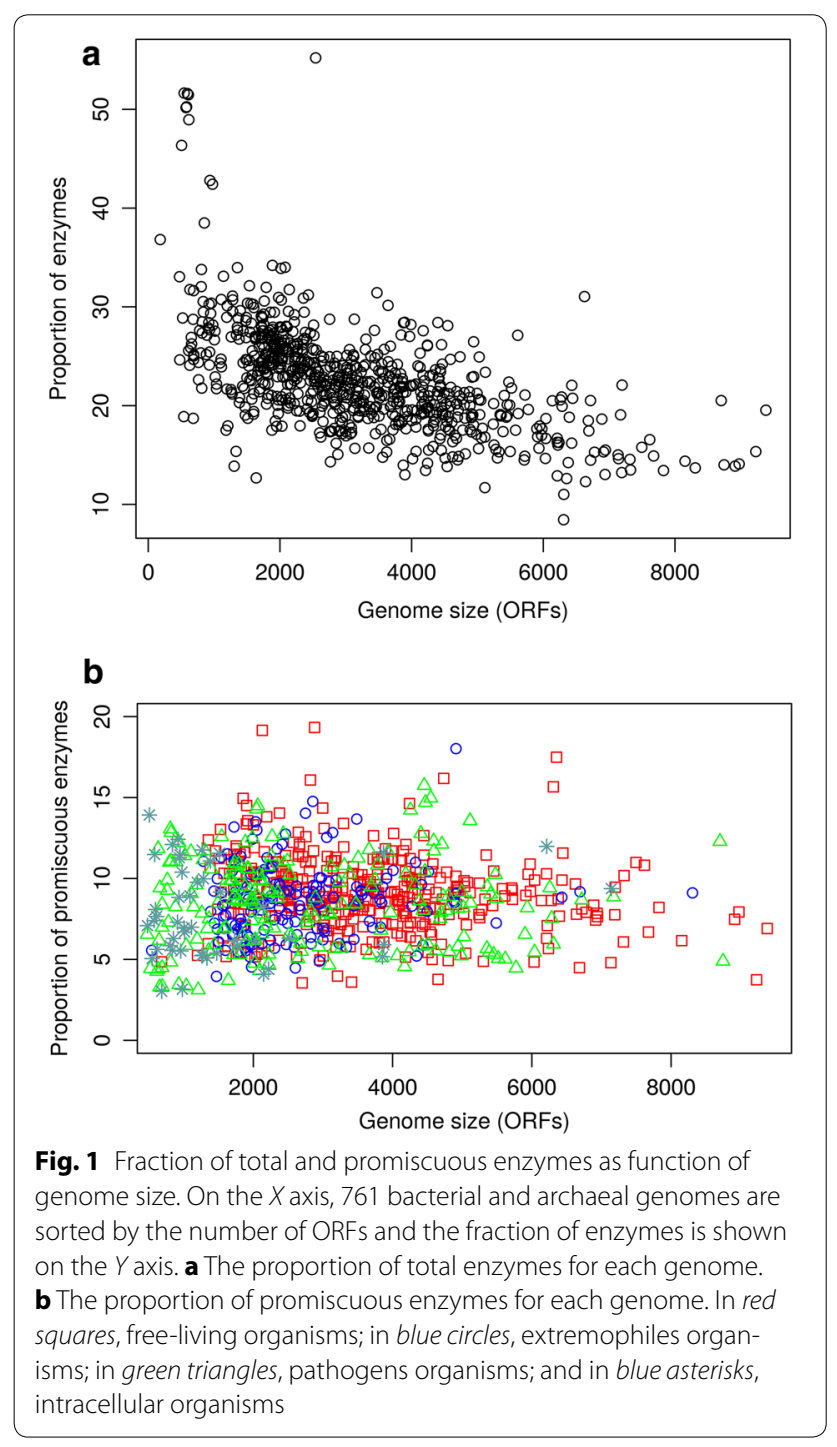

such as the appearance of novel substrates in the biochemical niche in which enzyme activity is expressed may influence the selective advantage that a new activity could provide [21]. In this context, an example of differential evolution in promiscuous enzymes associated to lifestyle is the secondary $N$-acyl amino acid racemase activity of the o-succinylbenzoate synthases from E. coli found in the gastrointestinal tract of animals, and in the soil bacterium Amycolaptosis sp. which differ by more than four orders of magnitude in their catalytic efficiency $[22,23]$.

In this regard, the ecological conditions would affect the repertoire of promiscuous enzymes, whose distribution of their fraction is different between the lifestyles of microorganism when Wilcoxon's test is applied ( $\mathrm{P}$ value $=0.01)$, [20]; for instance, free-living organisms exhibit a high number of promiscuous enzymes, followed by pathogenic, extremophiles and intracellular organisms (Fig. 2). The highest number of promiscuous enzymes identified in free-living organisms may be the consequence of an adaptation mechanism to survive in fluctuating ecological environments, such as in the Deinococcus-Thermus phylum which are highly resistant to environmental hazards [13]. The presence of a large proportion of promiscuous enzymes would allow the establishment of internal metabolic fluxes that can vary depending on environmental conditions, coupled with lower regulating promiscuous enzymes which enable rapid reprogramming of metabolic response, i.e., promiscuous enzymes would be subjected to less metabolic regulation than specialist enzymes, as it was previously suggested in $E$. coli [2]. Moreover, promiscuous enzymes might endow the organisms with a selective advantage and genome plasticity [24], which can help to contend against fluctuating ecological niches, such as those faced by free-living microorganisms.

\section{The role of gene duplication events in the promiscuous enzymes}

Gene duplication has been described as an important source of raw material for the generation of new functions [25, 26]. In this context, Khersonsky et al. [27] suggest that, the divergence of new function can proceed via a 'generalist' intermediate that exhibits broad specificity. Then, gene duplication may follow this process, rather than initiate it, and lead to divergence of a new 'specialist' enzyme. Thereby, a positive correlation between duplicated promiscuous enzymes and genome size has been

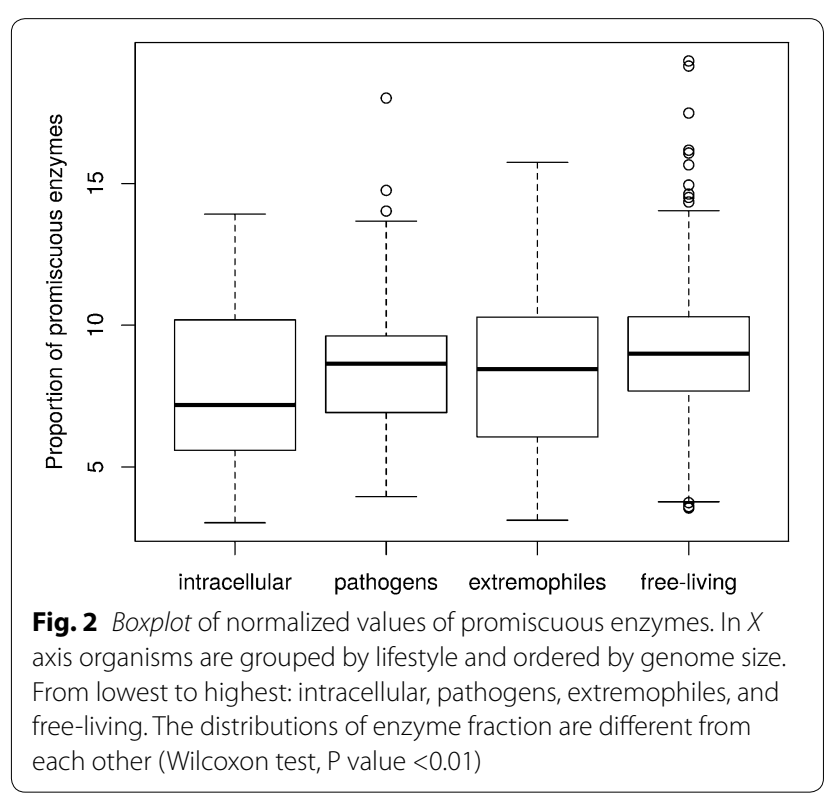


described [19] in which, the free-living organisms with larger genomes also have a greater number of duplicated genes encoding enzymes, while intracellular organisms have lower duplicated genes encoding enzymes as well as the smallest genomes. Thus, gene duplication events exhibit a high frequency in free-living and also in extremophilic organisms, wherein the percentage of promiscuous enzymes that arose by duplication in the genome is over $30 \%$ [20]. In contrast, intracellular organisms only contain $12 \%$ of promiscuous enzymes that have arisen by duplication, which is one third of that observed in freeliving and extremophiles. These findings suggest that duplication processes provide free-living and extremophilic organisms with raw material to improve the functions of proteins to contend and obtain nutrients from changing environments [19]. In contrast, in more stable environments, such as intracellular habitats, gene duplication events seem to be less significant because these organisms have been associated to massive gene loss [28], as consequence of their life-styles. The emergence and persistence of duplicated genes in organisms with fluctuating environments, promotes the improvement and innovation of proteins that can be used in nutrient assimilation of different compounds, which may be at low concentrations or in rare forms of assimilation. Finally, the diversification of functions as consequence of duplication promotes environmental adaptation of microorganisms.

\section{Conclusions}

A promiscuous function becomes important when a new carbon source is found or the existing one is limited, and therefore it is necessary its transformation to be useful for cell growth. Secondary activities of promiscuous enzymes are not under selective pressure, and they can accumulate mutations that decrease or increase their catalytic efficiency. The efficiency of promiscuous activities of enzymes may evolve in different degrees in the microbial species, depending on how the environment influences the evolutionary trajectories of enzymes [21] and the ability that promiscuity activity gives to each organism. Therefore, the original question raised in the title of this review, do lifestyles influence the presence of promiscuous enzymes in bacteria and Archaea metabolism?, seems to have a positive response. In this regard, although promiscuous enzymes appear uniformly distributed across all the species [20], there is enrichment in certain lifestyles, such as free-living organisms. Therefore, different evolutionary forces are acting on bacterial and archaeal metabolism; in the one hand, the abundance of total enzymes depends on genome size, while in the other hand, the abundance of promiscuous enzymes is influenced by the organism lifestyle. In this context, free-living and extremophilic organisms are enriched of promiscuous enzymes, perhaps as an adaptive mechanism, which is favored in species living in fluctuating environments. Evidences suggesting that extremophilic organisms are widely distributed in a variety of environments [29] reinforce the notion that the enrichment of promiscuous enzymes arise in fluctuating environments.

Finally, environment favors the appearance of promiscuous enzymes in species inhabiting fluctuating environments, as well as favors duplication events that allow functional divergence in enzymes. Thus, it seems that gene duplications occur more frequently in free-living organisms, where a third of their promiscuous enzymes have arisen by duplication. In contrast, organisms inhabiting more stable environments such as intracellular species have a lower proportion of duplicated and promiscuous genes encoding enzymes.

\section{Author details}

1 Laboratorio de Ecogenómica, Unidad Académica de Ciencias y Tecnología, Facultad de Ciencias, Universidad Nacional Autónoma de México, Mérida, Yucatán, Mexico. ${ }^{2}$ Departamento de Ingeniería Celular y Biocatálisis, Instituto de Biotecnología, Universidad Nacional Autónoma de México, Cuernavaca, Morelos, Mexico.

\section{Authors' contribution}

MAMN conceived the idea. MAMN design and performed the experiments. MANM, EPR analyzed the data. MANM, EPR wrote the paper. Both authors read and approved the final manuscript.

\section{Acknowledgements}

We would like to thank to Dr. Miguel Cocotl Yañez, Jose Antonio Ibarra, Maiana Oliveira and Dagoberto Armenta for their comments and suggestions to this manuscript. MAMN acknowledges the support of Cátedras CONACyT. EP-R was supported by a Grant (IN-204714) from DGAPA, UNAM.

\section{Competing interests}

The authors declare that they have no competing interests.

Received: 9 October 2015 Accepted: 7 February 2016

Published online: 16 February 2016

\section{References}

1. Cheng XY, Huang WJ, Hu SC, Zhang HL, Wang H, Zhang JX et al (2012) A global characterization and identification of multifunctional enzymes. PloS ONE 7(6):e38979. doi:10.1371/journal.pone.0038979

2. Nam H, Lewis NE, Lerman JA, Lee DH, Chang RL, Kim D et al (2012) Network context and selection in the evolution to enzyme specificity. Science. 337(6098):1101-1104

3. Huberts DH, van der Klei IJ (2010) Moonlighting proteins: an intriguing mode of multitasking. Biochim Biophys Acta 1803(4):520-525. doi:10.1016/j.bbamcr.2010.01.022

4. Hult K, Berglund P (2007) Enzyme promiscuity: mechanism and applications. Trends Biotechnol 25(5):231-238. doi:10.1016/j.tibtech.2007.03.002

5. Sengupta S, Ghosh S, Nagaraja V (2008) Moonlighting function of glutamate racemase from Mycobacterium tuberculosis: racemization and DNA gyrase inhibition are two independent activities of the enzyme. Microbiology. 154(Pt 9):2796-2803. doi:10.1099/mic.0.2008/020933-0

6. Jia B, Cheong GW, Zhang S (2013) Multifunctional enzymes in Archaea: promiscuity and moonlight. Extremophiles 17(2):193-203. doi:10.1007/ s00792-012-0509-1 
7. Jeffery CJ (2014) An introduction to protein moonlighting. Biochem Soc Trans 42(6):1679-1683. doi:10.1042/BST20140226

8. Kainulainen $V$, Korhonen TK (2014) Dancing to another tune-adhesive moonlighting proteins in bacteria. Biology. 3(1):178-204. doi:10.3390/ biology3010178

9. Choi-Rhee E, Schulman H, Cronan JE (2004) Promiscuous protein biotinylation by Escherichia coli biotin protein ligase. Protein Sci 13(11):30433050. doi:10.1110/ps.04911804

10. Khersonsky O, Tawfik DS (2010) Enzyme promiscuity: a mechanistic and evolutionary perspective. Annu Rev Biochem 79:471-505. doi:10.1146/ annurev-biochem-030409-143718

11. Copley SD (2015) An evolutionary biochemist's perspective on promiscuity. Trends Biochem Sci 40(2):72-78. doi:10.1016/j.tibs.2014.12.004

12. Bornscheuer UT, Kazlauskas RJ (2004) Catalytic promiscuity in biocatalysis: using old enzymes to form new bonds and follow new pathways. Angew Chem 43(45):6032-6040. doi:10.1002/anie.200460416

13. Carbonell P, Lecointre G, Faulon JL (2011) Origins of specificity and promiscuity in metabolic networks. J Biol Chem 286(51):43994-44004. doi:10.1074/jbc.M111.274050

14. Yip SH, Matsumura I (2013) Substrate ambiguous enzymes within the Escherichia coli proteome offer different evolutionary solutions to the same problem. Mol Biol Evol 30(9):2001-2012. doi:10.1093/molbev/ mst105

15. Kim J, Kershner JP, Novikov Y, Shoemaker RK, Copley SD (2010) Three serendipitous pathways in E. coli can bypass a block in pyridoxal-5'phosphate synthesis. Mol Syst Biol 6:436. doi:10.1038/msb.2010.88

16. Babtie AC, Bandyopadhyay S, Olguin LF, Hollfelder F (2009) Efficient catalytic promiscuity for chemically distinct reactions. Angew Chem 48(20):3692-3694. doi:10.1002/anie.200805843

17. Nobeli I, Favia AD, Thornton JM (2009) Protein promiscuity and its implications for biotechnology. Nat Biotechnol 27(2):157-167. doi:10.1038/ nbt1519

18. Arora B, Mukherjee J, Gupta MN (2014) Enzyme promiscuity: using the dark side of enzyme specificity in white biotechnology. Sustain Chem Process 2:1-9. doi:10.1186/s40508-014-0025-y
19. Martinez-Nunez MA, Poot-Hernandez AC, Rodriguez-Vazquez K, Perez Rueda E (2013) Increments and duplication events of enzymes and transcription factors influence metabolic and regulatory diversity in prokaryotes. PloS ONE 8(7):e69707. doi:10.1371/journal.pone.0069707

20. Martinez-Nunez MA, Rodriguez-Vazquez K, Perez-Rueda E (2015) The lifestyle of prokaryotic organisms influences the repertoire of promiscuous enzymes. Proteins 83(9):1625-1631. doi:10.1002/prot.24847

21. Kurtovic S, Shokeer A, Mannervik B (2008) Emergence of novel enzyme quasi-species depends on the substrate matrix. J Mol Biol 382(1):136-153. doi:10.1016/j.jmb.2008.07.003

22. Khanal A, Yu McLoughlin S, Kershner JP, Copley SD (2015) Differential effects of a mutation on the normal and promiscuous activities of orthologs: implications for natural and directed evolution. Mol Biol Evol 32(1):100-108. doi:10.1093/molbev/msu271

23. Palmer DR, Garrett JB, Sharma V, Meganathan R, Babbitt PC, Gerlt JA (1999) Unexpected divergence of enzyme function and sequence: "N-acylamino acid racemase" is o-succinylbenzoate synthase. Biochemistry. 38(14):4252-4258. doi:10.1021/bi990140p

24. Aharoni A, Gaidukov L, Khersonsky O, Mc QGS, Roodveldt C, Tawfik DS (2005) The evolvability of promiscuous protein functions. Nat Genet 37(1):73-76. doi:10.1038/ng1482

25. Martinez-Nunez MA, Perez-Rueda E, Gutierrez-Rios RM, Merino E (2010) New insights into the regulatory networks of paralogous genes in bacteria. Microbiology. 156(Pt 1):14-22. doi:10.1099/mic.0.033266-0

26. Ohno R (1970) Evolution by gene duplication. Springer, Heidelberg

27. Khersonsky O, Roodveldt C, Tawfik DS (2006) Enzyme promiscuity: evolutionary and mechanistic aspects. Curr Opin Chem Biol 10(5):498-508. doi:10.1016/j.cbpa.2006.08.011

28. Koskiniemi S, Sun S, Berg OG, Andersson DI (2012) Selection-driven gene loss in bacteria. PLoS Genet 8(6):e1002787. doi:10.1371/journal. pgen.1002787

29. Aller JY, Kemp PF (2008) Are Archaea inherently less diverse than bacteria in the same environments? FEMS Microbiol Ecol 65(1):74-87. doi:10.1111/j.1574-6941.2008.00498.x

\section{Submit your manuscript to a SpringerOpen ${ }^{\circ}$ journal and benefit from:}

- Convenient online submission

- Rigorous peer review

- Immediate publication on acceptance

- Open access: articles freely available online

- High visibility within the field

- Retaining the copyright to your article

Submit your next manuscript at $>$ springeropen.com 